\title{
Impact of COVID-19 on Out-Patient Services - Physiotherapy's Perspectives
}

\author{
Priya Sirohi ${ }^{1}$ \\ ${ }^{1}$ Centre for Physiotherapy and Rehabilitation Sciences, \\ Jamia Millia Islamia, New Delhi, India.
}

The rapid spread of Coronavirus disease (COVID-19) has led to global pandemic affecting mental and physical well-being of people around the world. Given the highly contagious nature of COVID-19, public authorities in order to restrict the escalation of the virus ordered social lockdown and asked people to self-quarantine at homes if they develop any symptoms. As a result, these new social regulations have changed how individuals live and carry out everyday tasks in the general society. Reduced physical activity during lockdown has enhanced vulnerability to develop infections and non-communicable diseases. While on the other side, due to absence of routine medical surveillance, the condition of people with existing chronic conditions has deteriorated. Re-allocation of health care resources to treat COVID-19 patients put forward unprecedented challenge for the healthcare system in terms of providing medical support to non-infected out-patients. Extended social lockdowns are also causing adverse repercussions for physiotherapy and rehabilitation out-patient services. COVID-19 restrictions have hampered the in-person interaction of therapists and patients for in-person consultations and therapy sessions. Thus, leading to overall delay in the management and recovery of patients, especially those who are at a critical stage of treatment. During these unprecedented times of COVID19 pandemic, provision of telehealth appeared to be the guiding light to tackle these evolving challenges of public health. Focusing on the out-patients who need close supervision for therapies and counselling, in this piece, "I" am arguing that methods of telemedicine and tele-rehabilitation should be actively adopted in the healthcare services to bridge the gap caused due to COVID-19.

\section{KEY WORDS}

COVID-19 Pandemic, Physical Activity, Healthcare, Out-Patient Services, Physiotherapy, Tele-Rehabilitation
Corresponding Author:

Priya Sirohi.

Student, Master of Physiotherapy (Sports),

Centre for Physiotherapy and

Rehabilitation Sciences, Jamia Millia

Islamia, New Delhi, India.

E-mail: ps.priyasirohi@gmail.com

DOI: $10.14260 / \mathrm{jemds} / 2020 / 721$

How to Cite This Article:

Sirohi P. Impact of COVID-19 on out-patient services: physiotherapy's perspectives. J Evolution Med Dent Sci 2020;9(44):32803283, DOI: $10.14260 /$ jemds/2020/721

Submission 28-07-2020,

Peer Review 22-09-2020,

Acceptance 28-09-2020,

Published 02-11-2020.

Copyright (c) 2020 Priya Sirohi. This is an open access article distributed under Creative Commons Attribution License [Attribution 4.0 International (CC BY 4.0)] 
Coronavirus disease 2019 (COVID-19) caused by Severe Acute Respiratory Syndrome Coronavirus 2 (SARS-CoV-2) is a novel illness declared as a pandemic by the World Health Organization (WHO) in March $2020 .{ }^{1}$ It has severely impacted the lives of people across the world. In India, the impact of the COVID-19 pandemic has been worse due to underlying issues of fragmented health infrastructure in semi-urban and rural parts of the country and due to weak public health governance. The Government of India has opted various measures to control the spread of COVID-19 and is responding to the needs of both COVID-19 patients and common people. Among other strategies, lockdown and social isolation are one of the most popular methods adopted by the government to control the viral spread. However, prolonged social isolation can also have severe impacts on people's physical and mental health and these impacts have already been started to be visible in general discourse. ${ }^{2}$

In the present COVID-19 induced pandemic, patients who need medical treatment for chronic illnesses or need supervision and consultation for therapy treatments have been affected severely. The public regulation has stipulated that medical support shall be provided on priority basis to urgent and critical patients only, leaving behind the other patients who need physical therapy and rehabilitation services in the limbo. However, the benefits of physical therapy have also been accepted widely for the rehabilitation of the patients recovering from COVID-19. ${ }^{3}$

To deal with the emerging issues of public health, prospects of telehealth and digital medical services are gradually being adopted by the health care sector in order to compensate for in-person medical consultation, keeping in mind, the quality of care as transparent and patient-centric as possible. However, there are certain challenges which need to be overcome for smooth implementation and delivery of digital care.

\section{Impact of Lockdown on Individual's Health}

In order to prevent the spread of virus, social lockdown has been implemented as a preventive measure to restrict physical contacts between individuals and by means of advising people to stay at home. However, staying indoor for extended periods with bare minimum outdoor / physical activities has been causing severe distress among the population, including on healthy people. The work routines during lockdown have also been drastically changed with decreased physical activities and increased time spent in sitting in sedentary positions. In the ongoing pandemic, while people are adjusting to new methods of work and adapting to new routines in daily life; they are also predisposed to develop health issues like weight gain, disturbed sleep cycles and musculoskeletal conditions among others. Social distancing is affecting people's health severely.

Physical activity and exercise are critical for keeping up good health and well-being and are also central for increasing immunity to fight against communicable and / or noncommunicable diseases (NCDs). Mobilization of various immune cells, including CD8+ T cells, neutrophils, and natural killer cells in response to physical activity 4 boosts immunesurveillance. Evidence from epidemiological studies has demonstrated the reduced incidence and mortality for upper respiratory tract infections like influenza linked to a physically active lifestyle and proper hygiene practices. ${ }^{5}$ Conversely, sedentary behaviour for a prolonged period of time is associated with an increment in systemic inflammation in conjunction with decreased cytokine production, T-cell proliferation ${ }^{6,7}$ leading to an immuno-compromised state to control the spread of pathogens.

The distress on people's health due to prolonged social isolation is also evident. Due to reduced or minimum physical work and movement, people are also at the high risk of being afflicted by arthralgia, myofascial pain, auto-immune disorders like spondylitis, systemic lupus erythematous, rheumatic arthritis. ${ }^{8}$ While the elderly population with existing chronic condition of disabling and painful osteoarthritis (of either single or multiple joints) which is by far the most prevalent musculoskeletal disorder affecting this age group are at the highest risk of infection with existing health issues of asthma, diabetes and heart disease as compared to age and gender-matched healthy individuals. ${ }^{9}$ It is imperative to note that lockdown and social isolation has serious effects on people's physical and mental well-being irrespective of their age, sex, and pre-health conditions.

\section{Physiotherapy and Other Out-Patient Services in Coronavirus Pandemic}

In India, the COVID-19 pandemic has knocked the health institutions, at all levels of its machinery, like never before. In several countries, including India, the secondary and tertiary care centres - which were earlier delivering services on an average to millions of out-patients, required critical transformation. The secondary and tertiary care centre has now been turned into facilities of temporary COVID-19 hospitals for treating patients with moderate to severe symptoms of the disease.

In a study, it has been indicated that the out-patient services at Primary Health Centres (PHCs) in India is constrained during the ongoing COVID-19 pandemic due to absence of required infrastructure and resources, and weak policy framework to deal with infection control and patient safety. ${ }^{10}$ Amid the pandemic, the condition is continuously worsening due to long shift hours and shortage of Personal Protective Equipment (PPE) for health workers. ${ }^{11}$ On one hand, while allocation of safety gears have been on priority for frontline health care workers - who are directly engaged in treating COVID-19 patients - on the other hand, the primary health care providers are left with limited resources which has increased their vulnerability.

Health care services managing NCDs have been widely afflicted around the globe during the lockdown. As per WHO's survey report, in which 155 nations took part, $94 \%$ of countries have partially or wholly reassigned healthcare providers managing NCDs towards the public health emergency of COVID-19. Around $53 \%$ of countries have partial to full disruption of health services for treating hypertension; $49 \%$ for management of diabetes and associated complications; $42 \%$ for cancer treatment and $31 \%$ for cardiovascular emergencies. ${ }^{12}$

Physiotherapy being a domain of health care practice dealing with rehabilitation of musculoskeletal, cardiopulmonary, neurological conditions have an objective to improve patients' muscle and joint functions, enhancing functional mobility and overall conditioning. Unavailability of 
in-person supervision of physical therapies due to social restrictions has increased the vulnerability of patients. Variety of patient population extending from paediatric, geriatric, neuropsychiatric, differently abled among others who were undergoing routine sessions of outpatient or home-based rehabilitation therapies requiring continuous supervision of physiotherapist for assessment, treatment and follow-ups are the most affected groups of patients. Problems are further deteriorated with absence and shortage of caregivers in addition to unavailability and inaccessibility to telehealth. It has also been reported that despite acting as a critical element to healthy recovery following COVID-19, rehabilitation services have been disrupted in $63 \%$ of the countries ${ }^{12}$ which in turn is increasing the overall burden of disease.

Physiotherapists, as health care providers, are also at higher risk of exposed to infection due to the usual nature of practice requiring close proximity for assessing the patients along with implementing various manual techniques which have to be done passively by the therapists themselves. Moreover, the risk is further enhanced if the physiotherapist health care provider is dealing with asymptomatic patients who are found to be transmitting the infection. ${ }^{13}$ If not taken care of, this may generate a chain of transmission from asymptomatic patients to therapists and other patients who may or may not have existing comorbidities, increasing the risk of spreading of the infection.

\section{Prospects of Tele-Rehabilitation and Exercise at Home}

'Telehealth' is an umbrella term used to explain the provision of healthcare services through Information and Communication Technologies (ICT). ${ }^{14}$ Though telehealth encompasses all health care domains; however, the term used to refer utilization of ICTs to deliver physical and rehabilitation therapy treatments and counselling is knows as tele-rehabilitation services. ${ }^{15}$ With the emergence of the pandemic, there has been as increase in patient's reliance on telemedicine and tele-rehabilitation for consultation and counselling.

Telehealth empowers healthcare delivery by means of telecommunication, leaving behind the conventional methods of in-person interaction. The mode of communication utilised in telehealth can be through written, audio, audio-visual or, internet-based methods. Telehealth aids patient to take benefits of various healthcare services which include taking history, modified physical examination, diagnostic testing, assessment and management. ${ }^{16}$ In the current scenario, telehealth is providing additional services such as quick diagnosis and consequent management and stabilisation of patients; close follow-ups; education of citizens; training of health providers; coordination with medical staff at remote locations; real-data surveillance across the world. This has also assists in cutting down the overall cost of disposal of protective gears and disinfection, imparting the green impact of telehealth. ${ }^{17}$

Efficacy of patient assessment and treatment via digital practice has been explored by various researchers for pain management, cardiac problems, respiratory dysfunctions, pelvic floor conditions and post-operative rehabilitation. ${ }^{18-21}$ The literature on the subject has outlined a high level of patient satisfaction to tele-rehabilitation services in musculoskeletal physiotherapy as well.22-23 Despite its tremendous benefits, uptake of telemedicine has been slow in India compared to other countries. The alternatives of telemedicine or telerehabilitation have been explored by practitioners to address the crisis caused by COVID-19 social restrictions. However, unavailability or inaccessibility of required ICTs' infrastructure besides the lack of expertise and experience of both professionals to deliver the services and patients to take benefits of ICT induced services is hampering the adoption of digital medical services.

However, in order to maintain active physical health, there are few physical activities and exercises that people can carry out either independently or with the help of mobile applications to keep them physically active. (Table 1)

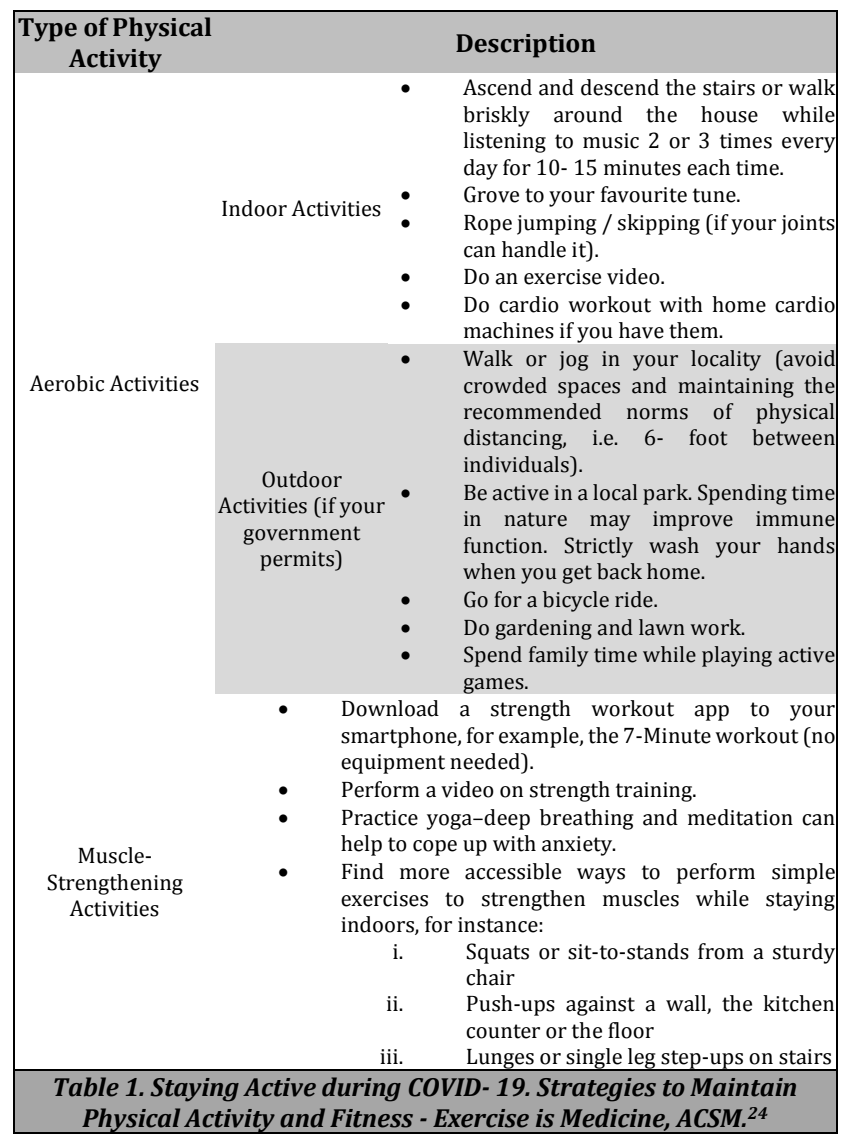

\section{CONCLUSIONS}

COVID-19 pandemic is considered to be one of the biggest challenges faced by humankind. Majority of nations has adopted the model of social lockdown, limited social mobility while promoting mandatory social-distancing to prevent and control the rapid progression of the disease. However, extended periods of self-quarantine have influenced the physical, mental, social and economic wellness of people across the globe. During the social lockdown, increased physical inactivity, and sedentary behaviour have led to lifestyle-related disorders among healthy individuals. On the other hand, people with existing chronic illnesses have been severely affected due to the lack of routine medical supervision and follow-ups.

Out-patient departments, including physiotherapy and rehabilitation services, are not spared by the ongoing 
pandemic. There has been a considerable impact on guided, regular sessions of exercise therapies and rehabilitation. However, this led patients to seek medical care via digital health services or, telemedicine. Since the practice of telehealth is new to various nations alongside the lack of required infrastructure, it is challenging to deliver and receive the benefits of these services in such a scenario.

At present, the focus of the public health sector is concentrated to test, detect, and treat the COVID-19 patients. However, besides this, it is equally important to boost digital healthcare infrastructure along with training of professionals to provide access to health services through digital platforms to avoid a parallel health crisis. If it goes unnoticed, it will increase the burden on the health institutions and would further deteriorate the public health systems. Additionally, it is also essential for healthcare professionals to learn about the proper use of PPE, infection control methods, waste management and environmental cleaning and disinfection in order to decrease the risk of contagion in both, hospital and clinical settings. People, on the other hand, need to incorporate regular physical activity and exercises in their daily routine, keeping in mind the norms of social distancing and staying at home for a healthier and active lifestyle.

Financial or other competing interests: None.

Disclosure forms provided by the authors are available with the full text of this article at jemds.com.

\section{REFERENCES}

[1] WHO Director-General's opening remarks at the media briefing on COVID-19. 11 March 2020. https://www.who.int/speeches/detail/who-directorgeneral-s-opening-remarks-at-the-media-breifingoncovid-19---11-march-2020

[2] Zhang SX, Wang Y, Rauch A, et al. Unprecedented disruption of lives and work: health, distress and life satisfaction of working adults in China one month into the COVID-19 outbreak. Psychiatry Res 2020;288:112958.

[3] Yu P, Wei Q, He C. Early rehabilitation for critically ill patients with COVID-19: more benefits than risks. Am J Phys Med Rehabil 2020;99(6):468-9.

[4] Simpson RJ, Kunz H, Agha N, et al. Exercise and the regulation of immune functions. Prog Mol Biol Transl Sci 2015; 135:355-80.

[5] Wu S, Ma C, Yang Z, et al. Hygiene behaviors associated with influenza-like illness among adults in Beijing, China: a large, population-based survey. PLoS One 2016;11(2):e0148448.

[6] Murdaca G, Setti M, Brenci S, et al. Modifications of immunological and neuro-endocrine parameters induced by antiorthostatic bed-rest in human healthy volunteers. Minerva Med 2003;94(6):363-78.

[7] Uchakin PN, Stowe RP, Paddon-Jones D, et al. Cytokine secretion and latent herpes virus reactivation with 28 days of horizontal hypokinesia. Aviat Space Environ Med 2007;78(6):608-12.

[8] Silva RMV, Sousa AVC. Chronic phase of COVID-19: challenges for physical therapists in the face of musculoskeletal disorders. Fisioterapia em Movimento 2020;33:e0033002.
[9] Marks R, Allegrante JP. Comorbid disease profiles of adults with end-stage hip osteoarthritis. Med Sci Monit 2002;8(4):CR305-9.

[10] Garg S, Basu S, Rustagi R, et al. Primary health care facility preparedness for outpatient service provision during the COVID-19 pandemic in india: cross-sectional study. JMIR Public Health Surveill 2020;6(2):e19927.

[11] O'Sullivan ED. PPE guidance for COVID-19: be honest about resource shortages. BMJ 2020;369:m1507.

[12] COVID-19 significantly impacts health services for noncommunicable diseases. https://www.who.int/newsroom/detail/01-06-2020-covid-19-significantly-impactshealth-services-for-noncommunicable-diseases

[13] Rothe C, Schunk M, Sothmann P, et al. Transmission of 2019-nCoV infection from an asymptomatic contact in Germany. N Engl J Med 2020;382(10):970-1.

[14] Darkins A, Cary MA. Telemedicine and telehealth: principles, policies, performances and pitfalls. Springer Publishing Company 2000.

[15] Brennan D, Tindall L, Theodoros D, et al. A blueprint for telerehabilitation guidelines. Int $\mathrm{J}$ Telerehabil 2010;2(2):31-4.

[16] Tenforde AS, Hefner JE, Kodish-Wachs JE, et al. Telehealth in physical medicine and rehabilitation: a narrative review. PM\&R 2017;9(5):S51-8.

[17] Vidal-Alaball J, Acosta-Roja R, Hernández NP, et al. Telemedicine in the face of the COVID-19 pandemic. Aten Primaria. 2020;52(6):418-22.

[18] Kruse CS, Karem P, Shifflett K, et al. Evaluating barriers to adopting telemedicine worldwide: a systematic review. J Telemed Telecare 2018;24(1):4-12.

[19] Kitsiou S, Paré G, Jaana M. Effects of home telemonitoring interventions on patients with chronic heart failure: an overview of systematic reviews. J Med Internet Res 2015;17(3):e63.

[20] Hanlon P, Daines L, Campbell C, et al. Telehealth interventions to support self-management of long-term conditions: a systematic metareview of diabetes, heart failure, asthma, chronic obstructive pulmonary disease, and cancer. J Med Internet Res 2017;19(5):e172.

[21] Sjöström M, Umefjord G, Stenlund H, et al. Internet-based treatment of stress urinary incontinence: a randomised controlled study with focus on pelvic floor muscle training. BJU Int 2013;112(3):362-72.

[22] Moffet $H$, Tousignant $M$, Nadeau $S$, et al. Patient satisfaction with in-home telerehabilitation after total knee arthroplasty: results from a randomised controlled trial. Telemed J E Health 2017;23(2):80-7.

[23] Tousignant $M$, Boissy $P$, Moffet $H$, et al. Patients' satisfaction of healthcare services and perception with inhome telerehabilitation and physiotherapists' satisfaction toward technology for post-knee arthroplasty: an embedded study in a randomised trial. Telemed J E Health 2011;17(5):376-82.

[24] Staying active during COVID-19. https://www.exerciseismedicine.org/support_page.php/ stories $/ ? \mathrm{~b}=892$ 